\title{
Oxidación a alta temperatura de recubrimientos de CoNiCrAlY
}

\author{
F. J. BELZUNCE ${ }^{1}$, V. HIGUERA ${ }^{2}$ Y S. POVEDA ${ }^{2}$ \\ ${ }^{1}$ Dto. de Ciencia de Materiales, Universidad de Oviedo, Campus de Gijón, 33203 Gijón \\ ${ }^{2}$ Dto. de Energía, Universidad de Oviedo, Campus de Gijón, 33203 Gijón
}

\begin{abstract}
Se ha estudiado el comportamiento frente a la oxidación al aire a alta temperatura de un recubrimiento de CoNiCrAlY proyectado por detonación de alta frecuencia. Las capas obtenidas tras la proyección térmica en la atmósfera ambiental tienen una compacidad y adherencia muy altas y resultan muy poco afectadas tras una oxidación cíclica al aire bajo temperaturas máximas de $1000^{\circ} \mathrm{C}$. En estas condiciones se mantiene el espesor de la capa protectora, dado que se genera espontáneamente una fina y compacta capa de alúmina, continua y de espesor homogéneo, que proporciona una protección eficaz contra la oxidación.

\section{Palabras clave: Proyección térmica. Proyección por detonación. Cinética de oxidación. Recubrimientos de MCrAlY. Protección frente a la oxidación}

\section{High temperature oxidation of CoNiCrAlY}

The high temperature oxidation behaviour in air of thermal sprayed CoNiCrAlY coatings obtained using a high frequency detonation gun was studied. The coatings have high cohesion and good substrate/coating adherence, being not significantly affected after cyclic oxidation in air under a maximum temperature of $1000^{\circ} \mathrm{C}$. The thickness of the coating is not modified after the oxidation tests, and a thin and compact layer of alumina with a uniform thickness is created and as a result a good oxidation protection has been obtained.
\end{abstract}

Keywords:Thermal projection. Detonation gun. Oxidation kinetics. MCrAlY coatings. Oxidation protection

\section{INTRODUCCION}

Actualmente se están fabricando ya unidades de generación de energía eléctrica de más de 200MW utilizando para ello turbinas de gas en ciclos energéticos combinados, que permiten obtener rendimientos superiores al $50 \%$, cuando el rendimiento de las turbinas de vapor se sitúa en torno al 30\%. Estas turbinas suelen utilizar gas natural o gas-oil como combustible y son más robustas y pesadas que las que se emplean en los motores aeronáuticos y también trabajan en condiciones más estables que aquellas y a revoluciones inferiores (sobre 3000 rpm), por lo que los esfuerzos dinámicos tienen en estos casos mucha menor importancia, siendo por el contrario la resistencia a la oxidación y a la corrosión a alta temperatura de los materiales utilizados en su construcción las exigencias imperativas en estos diseños $(1,2,3)$.

Las superaleaciones base niquel utilizadas en la fabricación de los álabes de los primeros escalones de las turbinas de gas no son suficientemente resistentes a la oxidación en atmósferas como las de los gases de combustión de las turbinas (altamente oxidantes y temperaturas iguales o superiores a $1000^{\circ} \mathrm{C}$ ), por lo que deben recubrirse necesariamente con capas del tipo MCrAlY (el metal $\mathrm{M}$ es normalmente $\mathrm{Ni}$, Co o ambos). Además, en el caso de una turbina de gas de una central eléctrica de ciclo combinado, debe pensarse que estas capas deben ser capaces de aguantar ciclos térmicos severos repetidos, sin sufrir daños importantes (resistencia a la fatiga térmica) y también, igualmente, situaciones de corrosión en caliente producidas por la presencia de impurezas en el combustible (vanadio o azufre) y en el aire de admisión (cloro, sodio, potasio) (4).

El contenido de cromo y aluminio de los recubrimientos debe ajustarse con el fín de formar, en las condiciones de trabajo, una capa protectora de $\mathrm{Al}_{2} \mathrm{O}_{3}$ o de $\mathrm{Cr}_{2} \mathrm{O}_{3}$, siendo la primera de ellas preferible en atmósferas oxidantes y la segunda si se desea prevenir la corrosión por debajo de $900^{\circ} \mathrm{C}$ (5). Por otro lado, la incorporación de un pequeño porcentaje de Ytrio mejora la adherencia de la capa protectora de alúmina (6). Las adiciones de otros elementos como el Hafnio, Platino, Titanio o Silicio también se han revelado satisfactorias en ciertas aplicaciones concretas (7).

La proyección térmica es normalmente la técnica utilizada para depositar las capas protectoras de MCrAlY, especialmente la proyección por plasma en condiciones de baja presión (LPPS), con objeto de conseguir una capa prácticamente sin poros ni óxidos. Sin embargo, hoy día, se están obteniendo ya estas capas con un grado de cohesión muy alto, empleando otros métodos más económicos y de mayor disponibilidad, como son la técnica HVOF o el cañon detonador. El espesor habitual de las capas de protección varía en las distintas aplicaciones entre 75 y $125 \mu \mathrm{m}$ (8).

\section{PROCEDIMIENTO EXPERIMENTAL}

Los recubrimientos antioxidantes se ejecutaron en la empresa Aerostar Coatings (Irún) utilizando la técnica de proyección por detonación de alta frecuencia HFPD, que nos asegura una capa muy compacta (con una porosidad muy baja) y una alta fuerza de anclaje (adherencia). Se ha utilizado como sustrato un acero inoxidable austenítico (tipo 310). Se emplearon probetas cilíndricas de $25 \mathrm{~mm}$ de diámetro, según norma ASTM 
C633 (9). La composición química y granulometría del polvo del recubrimiento utilizado se muestran en la Tabla I. La Figura 1 muestra el aspecto y la distribución granulométrica del polvo de CoNiCrAlY: se trata de un polvo con una morfología esferoidal que se ha obtenido por atomización gaseosa. Las condiciones de ejecución de la proyección se reflejan en la Tabla II.

Se utilizaron técnicas de microscopía óptica para determinar, tras la proyección, el espesor de las capas proyectadas, su microestructura, porosidad y grado de oxidación. La porosidad y el grado de oxidación se obtuvieron por recuento manual de puntos en un microscopio metalográfico Nikon. Se midió también la microdureza Vickers de la capa y la adherencia sustrato/capa según norma ASTM C633 (9). Finalmente se realizó un análisis difractométrico del recubrimiento recién proyectado en un difractómetro Philips PW 1729 utilizando ánodo de cobre.

Posteriormente se realizaron ensayos de oxidación cíclica a $1000 \pm 5^{\circ} \mathrm{C}$ en un horno de laboratorio para lo que se emplearon ciclos repetidos consistentes en un mantenimiento en el horno durante 8 horas seguido de un enfriamiento al aire (velocidad de enfriamiento media: $120^{\circ} \mathrm{C} / \mathrm{min}$ ). Estos ciclos se prolongaron hasta un máximo de 160 horas (20 ciclos) con objeto de determinar la cinética de la oxidación de estas capas, para lo que se realizaron mediciones gravimétricas y metalográficas. Se realizaron igualmente, como referencia, ensayos cíclicos de oxidación del acero inoxidable austenítico 310 (sustrato).

Tras los ensayos de oxidación, las probetas fueron niqueladas con el fín de preservar la capa de óxido y posteriormente analizadas mediante técnicas de microscopía óptica y microscopía electrónica de barrido. También se realizaron análisis difractométricos superficiales (DRX) con objeto de detectar variaciones en las diferentes fases que componen el recubrimiento.

\section{RESULTADOS}

La Tabla III muestra los resultados obtenidos en la caracterización del recubrimiento, efectuada después de la proyección térmica $(0 \mathrm{~h})$. Destacamos la baja porosidad obtenida y la excelente adherencia; sin embargo el grado de oxidación (oxidación interna) es algo alto en virtud de la fina granulometría del polvo utilizado y por haber realizado la proyección en la atmósfera ambiental. De cualquier manera, el sistema de proyección empleado exige utilizar partículas de polvo muy finas con objeto de lograr alcanzar en la proyección temperaturas próximas a su fusión y asegurar de este modo una alta compacidad de la capa.

La misma Tabla III muestra la variación de las características principales de la capa de CoNiCrAlY con el tiempo de permanencia a $1000^{\circ} \mathrm{C}$. Se hace notar que el espesor, porosidad y la adherencia apenas resultan modificados por el ciclado térmico realizado, a pesar de haber empleado velocidades de enfriamiento importantes. Sin embargo, se registra una fuerte oxidación interna ya al cabo de las primeras 8 horas, que luego se mantiene sin alteraciones significativas. Esta oxidación está ligada a la porosidad abierta que presentan las capas proyectadas térmicamente. Igualmente, la microdureza de la capa aumenta tras una oxidación de 8 horas y luego ya se mantiene con pocas variaciones. Por lo tanto, el incremento de dureza registrado está ligado a la oxidación interna del recubrimiento.

La Figura 2 muestra el espectro de difracción de rayos $X$ de las capas de CoNiCrAlY tras la proyección y después de un
TABla I. COMPOSICIÓN, GRANULOMETRÍA Y MORFOLOGÍA DEL POLVO

\begin{tabular}{|l|l|l|l|l|l|l|l|}
\hline Polvo & $\%$ Co & $\% \mathrm{Ni}$ & $\% \mathrm{Cr}$ & $\% \mathrm{Al}$ & $\% \mathrm{Y}$ & Granulometría & Morfología \\
\hline Amdry 9954 & Resto & 32 & 21 & 8 & 0.5 & $-62+11 \mathrm{~mm}$ & Esferoidal \\
\hline
\end{tabular}

TABla II. PARÁMetros de PROYECCIÓN

\begin{tabular}{|l|l|}
\hline Sistema de proyección & $\begin{array}{l}\text { HFPD modelo PK200. Pistola: } \mathbf{\phi 2 0 ~} \mathbf{~ m m} \\
\text { Longitud de cañón: 250 mm }\end{array}$ \\
\hline Caudal de oxígeno $($ slpm) & 110 \\
\hline Caudal de nitrógeno $(\mathrm{slpm})$ & 120 \\
\hline Caudal de gas natural $(\mathrm{slpm})$ & 80 \\
\hline Caudal gas portador, $\mathrm{N}_{2}(\mathrm{slpm})$ & 20 \\
\hline Frecuencia $(\mathrm{Hz})$ & 75 \\
\hline Distancia de proyección $(\mathrm{mm})$ & 150 \\
\hline Alimentación (Twin Sulzer, g/min) & 30 \\
\hline Velocidad de translación $(\mathrm{cm} / \mathrm{s})$ & 10 \\
\hline Desplazamiento entre pasadas $(\mathrm{mm})$ & 10 \\
\hline
\end{tabular}

TABLA III. CARACTERÍSTICAS GENERALES DE LA CAPA TRAS LA PROYECCIÓN Y SU VARIACIÓN CON EL TIEMPO DE OXIDACIÓN A $1000^{\circ} \mathrm{C}$

\begin{tabular}{|l|l|l|l|l|l|l|}
\hline & $0 \mathrm{~h}$ & $8 \mathrm{~h}$ & $16 \mathrm{~h}$ & $40 \mathrm{~h}$ & $80 \mathrm{~h}$ & $160 \mathrm{~h}$ \\
\hline $\begin{array}{l}\text { Espesor } \\
(\mu \mathrm{m})\end{array}$ & $175 \pm 25$ & $190 \pm 20$ & $175 \pm 20$ & $175 \pm 25$ & $160 \pm 25$ & $165 \pm 25$ \\
\hline Porosidad & $0.8 \pm 0.2$ & $1.2 \pm 0.3$ & $1.1 \pm 0.5$ & $1.0 \pm 0.3$ & $1.2 \pm 0.3$ & $1.6 \pm 0.4$ \\
\hline $\begin{array}{l}\text { Oxidación } \\
\text { interna }\end{array}$ & $1.4 \pm 0.5$ & $7.5 \pm 1$ & $10.5 \pm 1$ & $8 \pm 1$ & $7 \pm 1$ & $8 \pm 0.5$ \\
\hline $\mathrm{HV}$ & $320 \pm 30$ & $365 \pm 30$ & $350 \pm 30$ & $350 \pm 30$ & $360 \pm 30$ & $340 \pm 30$ \\
\hline $\begin{array}{l}\text { Adherencia } \\
(\mathrm{MPa})\end{array}$ & $>65$ & - & - & - & $>60$ & 55 \\
\hline
\end{tabular}

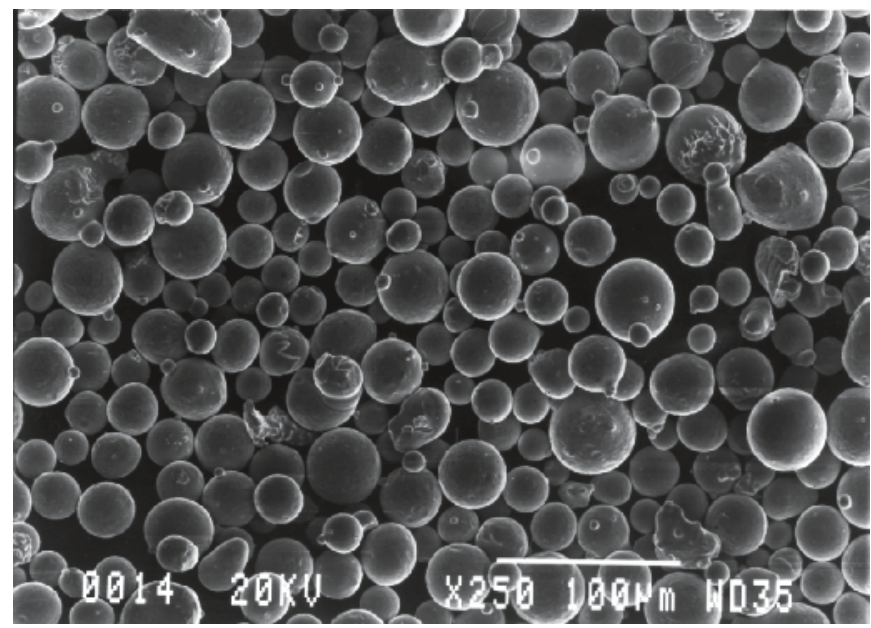

Figura 1. Morfología del polvo de CoNiCrAlY (Amdry 9954)

mantenimiento de $160 \mathrm{~h}$ a $1000^{\circ} \mathrm{C}$. Se observa la formación de alúmina $\left(\mathrm{Al}_{2} \mathrm{O}_{3}\right)$, así como de $\mathrm{CoAl}_{2} \mathrm{O}_{4}$. $\mathrm{El}$ aspecto general de la capa tras una oxidación a $1000^{\circ} \mathrm{C}$ prolongada durante 80 horas se puede observar en la Figura 3. Se pone de manifiesto el depósito de niquel (capa blanca) utilizado para no alterar la capa de óxido superficial (capa continua gris), así como un cierto grado de oxidación interna asociada a la porosidad intrínseca de la capa. La Figura 4 muestra ya con un mayor grado de detalle la capa oxidada superficial. Se trata de una capa continua de espesor muy homogéneo constituida por alúmina, aunque en su región más superficial, y con una morfología mucho más irregular, también se ha detectado, haciendo uso de la microsonda electrónica, la presencia de la espinela $\mathrm{CoAl}_{2} \mathrm{O}_{4}$. De cualquier manera, es la capa continua y com- 

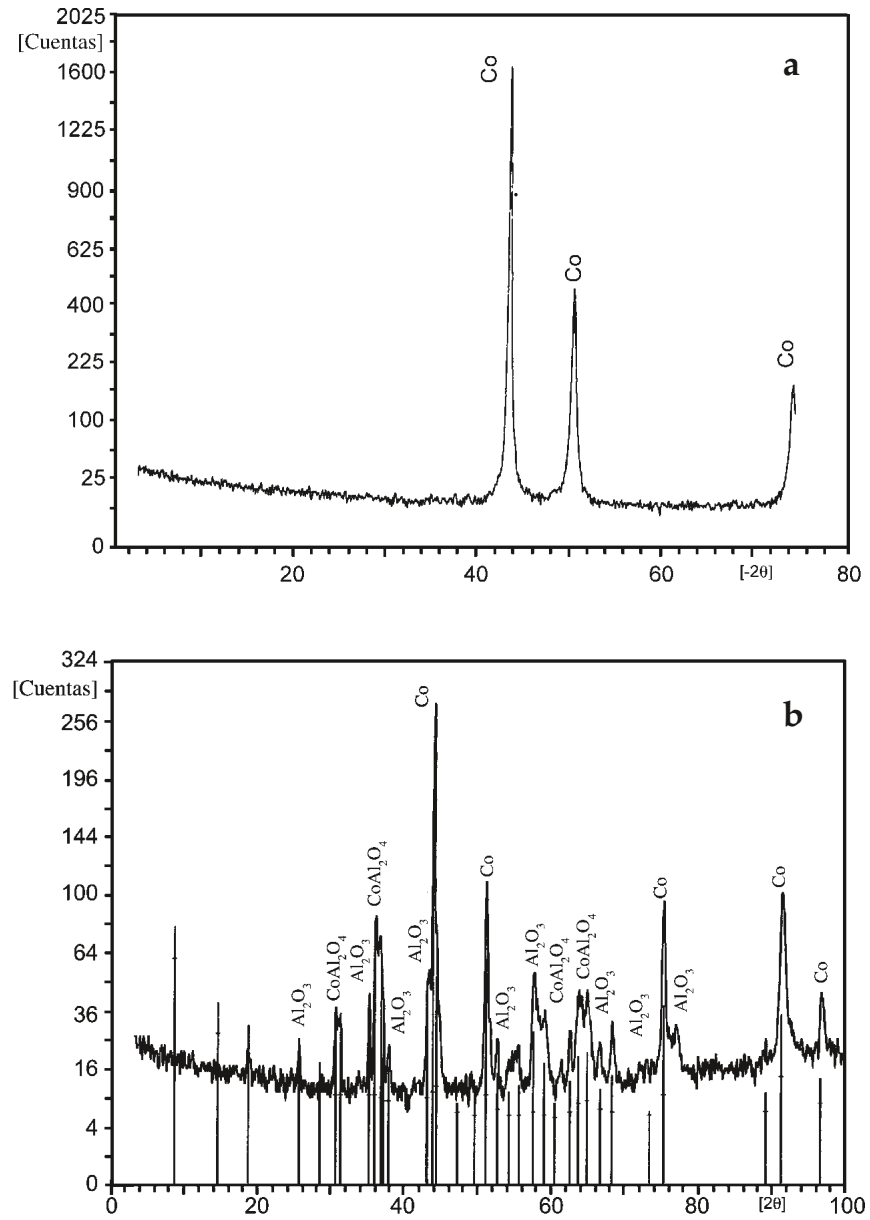

Figura 2. Espectros de difracción de rayos X. a) Capas de CoNiCrAlY tras la proyección b) Tras oxidación a $1000^{\circ} \mathrm{C}$ durante $160 \mathrm{~h}$.

pacta de alúmina la que actuando a modo de barrera, dificulta la difusión de los iones a su través y proporciona un buen comportamiento ante la oxidación.

La Figura 5 representa la variación total de peso por unidad de superficie que han experimentado en el curso de nuestos ensayos de oxidación cíclica las probetas recargadas con CoNiCrAlY. La ganancia de peso por unidad de superficie experimentada por los recubrimientos de CoNiCrAlY se ha calculado corrigiendo la ganancia global experimentada por la probeta con los datos recogidos de la oxidación del acero 310 para las misma condiciones de temperatura y tiempo. Por esta razón estos datos no deben usarse más que en términos cualitativos ya que a la temperatura de $1000^{\circ} \mathrm{C}$ el óxido $\mathrm{Cr}_{2} \mathrm{O}_{3}$, que se forma en la superficie del acero inoxidable 310, se oxida a $\mathrm{CrO}_{3}$, que es un óxido volatil a la temperatura de operación, lo que por un lado justifica la disminución de peso registrada en los tiempos de permanencia más largos, y por otro, puede introducir errores en la cuantificación del grado de oxidación del recubrimiento, ya que el resto de las superficies expuestas de las probetas utilizadas en los ensayos correspondían al acero 310 (10).

Por esta razón, se presenta finalmente en la Figura 6 la variación del espesor de la capa de alúmina (medida metalográfica-

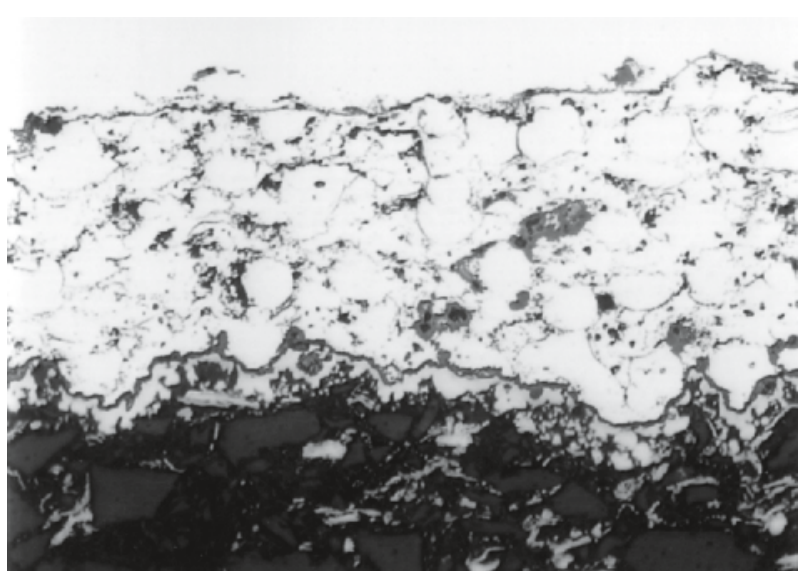

Figura 3. Capa de CoNiCrAlY tras 80 horas de oxidación a $1000^{\circ} \mathrm{C}$.

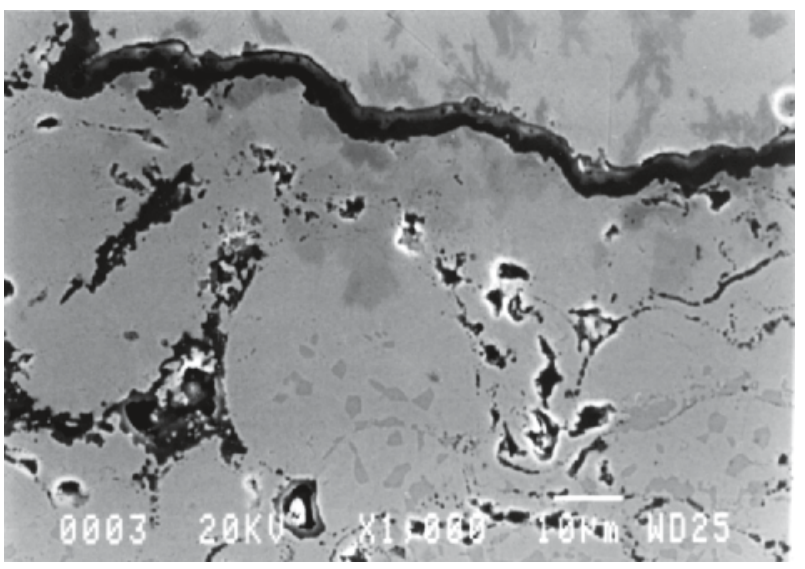

Figura 4. Capa superficial de $\mathrm{Al} 2 \mathrm{O} 3$ después de 160 horas a $1000^{\circ} \mathrm{C}$.

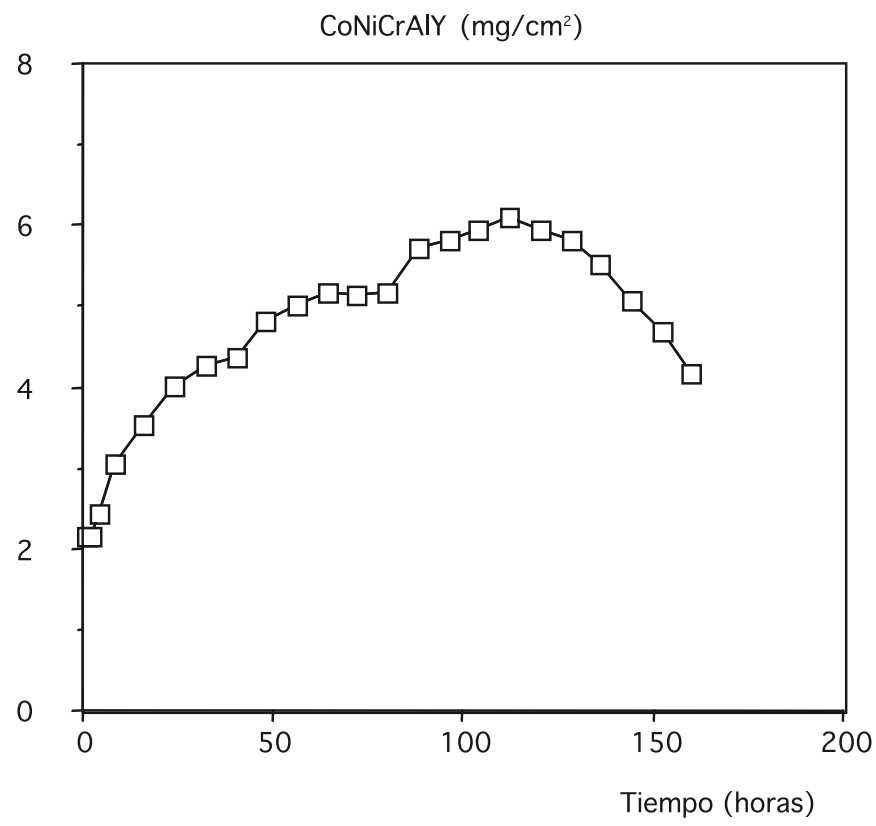

Figura 5. Ganancia de peso por unidad de superficie de las probetas recargadas con CoNiCrAlY. 
mente) con el tiempo de mantenimiento a $1000^{\circ} \mathrm{C}$. Como el espesor de la capa de alúmina es muy homogeneo (véase Figura 4), la medida se puede hacer con una buena precisión. Resulta así una ley cinética de oxidación de tipo logarítmico, que junto con el mantenimiento del espesor de capa, que se había constatado en estos ensayos (véase la Tabla III) dan cuenta del caracter protector de las capas de CoNiCrAlY en las condiciones de operación.

\section{CONCLUSIONES}

Las capas de aleación CoNiCrAlY proyectadas por detonación de alta frecuencia han mostrado una alto grado de cohesión, una adherencia capa/sustrato muy alta, aunque sin embargo la ejecución de la proyección en la atmósfera ambiente genera inevitablemente un cierto grado de oxidación, que no repercute desfavorablemente en la resistencia a la oxidación de este tipo de recubrimientos.

El comportamiento de estas capas protectoras ante ensayos de oxidación cíclicos a $1000^{\circ} \mathrm{C}$ al aire ha sido excelente. El espesor del recubrimiento no se ha modificado, la adherencia sustrato/ capa apenas se ha visto debilitada y la formación de una capa superficial de alúmina fina y continua, de espesor homogéneo y creciente con el tiempo de mantenimiento a alta temperatura proporciona una protección eficaz ante la oxidación al aire a alta temperatura.

\section{AGRADECIMIENTOS}

Los autores agradecen la financiación recibida del II Plan Regional de Investigación del Principado de Asturias para la ejecución del Proyecto de investigación PBMAT-9803. Igualmente destacan la participación de Aerostar Coatings en la ejecución de los recubrimientos empleados en el trabajo.

\section{BIBLIOGRAFÍA}

1. R. Chellini, The integrated gasification combined-cycle plant at Puertollano, Diesel\&Gas Turbines Worldwide (Octubre) 12-14, 1997.

2. B. Nilsson, GTX-100-A new high-performance gas turbine, ABB Review, Vol. 6, 4-12, (1997).

3. Czech N., Developments in materials for gas turbine blades, Siemens Power

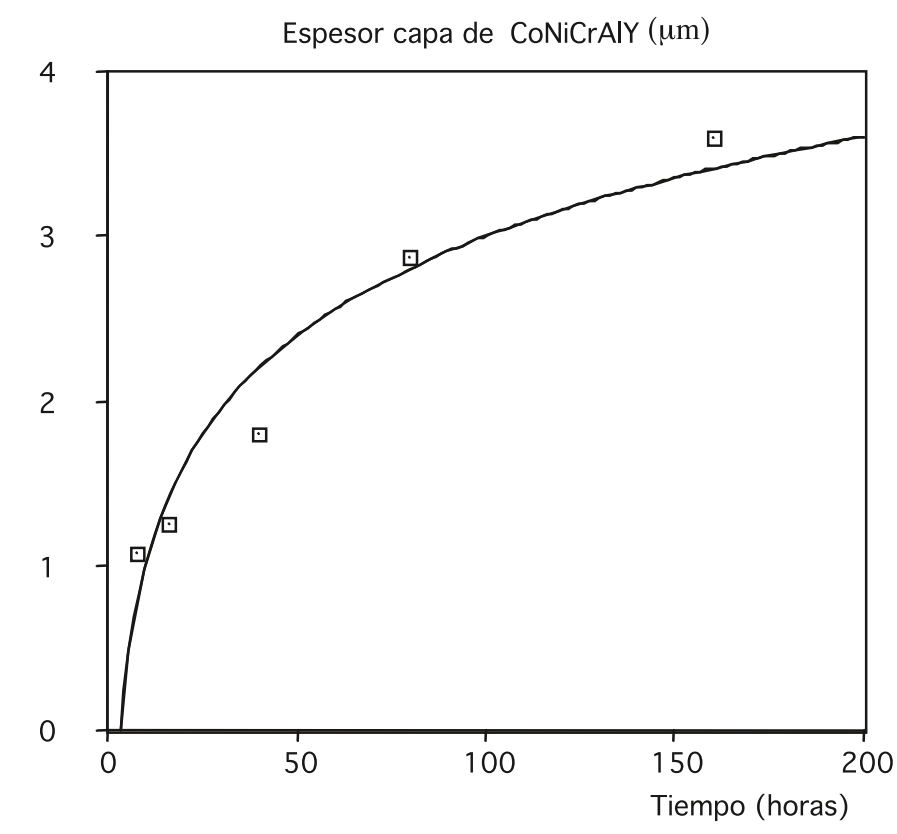

Figura 6. Evolución del espesor de la capa de alúmina con el tiempo de mantenimiento a $1000^{\circ} \mathrm{C}$ de las probetas de CoNiCrAlY.

Journal, 4, 22-27, (1994).

4. Parks W.P., Hoffman E.E., Lee W.Y. y Wright I.G., Thermal barrier coatings issues in advanced land-based gas turbines, J. Thermal Spray Tech., 6, 2, 187-192, (1997)

5. A.R. Nicoll et al., Future developments in plasma spray coatings, Mat. Scie. and Tech., Vol. 2 (Marzo), 214-219, 1986).

6. H.M.Tawancy, N.M.Abbas y A.Bennett, Role of Y during high temperature oxidation of MCrAlY coating on a Ni-base superalloy, Surf. and Coat. Tech., 68/69, 10-16, (1994)

7. G.W.Goward, Protective coatings-purpose, role and design, Mat. Scie. and Tech., Vol. 2 (Marzo) 194-200, 1986).

8. B.J.Gill y R.C.Tucker, Plasma spray coating processes, Mat. Scie. and Tech., Vol. 2 (Marzo) 207-213, 1986).

9. ASTM C633, Standard method for adhesion or cohesive strength of flamesprayed coatings, ASTM Standards (1976).

10. N. Birks y G.H.Meier, Introduction to high temperature oxidation of metals, Edward Arnold Pub., Reino Unido (1983). 